\title{
CsMnBr3: Lead-Free Nanocrystals with High Photoluminescence Quantum Yield and Picosecond Radiative Lifetime
}

Jawaher Almutlaq $^{\perp, \ddagger}$, Wasim J. Mir ${ }^{\perp, \ddagger}$, Luis Gutiérrez-Arzaluz ${ }^{\dagger, \perp}$, Jun Yin ${ }^{\dagger}{ }^{\perp}$, Serhii Vasylevskyi ${ }^{\S}$, Partha Maity ${ }^{\dagger}, \perp$, Jiakai Liu $^{\perp}$, Rounak Naphade ${ }^{\perp}$, Omar F. Mohammed $^{\dagger, \perp *}$, and Osman M. Bakr ${ }^{\perp *}$

$\perp$ King Abdullah University of Science and Technology (KAUST), KAUST Catalysis Center (KCC), Physical Science and Engineering (PSE) Division, Thuwal 23955-6900, Kingdom of Saudi Arabia.

$\uparrow$ King Abdullah University of Science and Technology (KAUST), Advanced Membranes and Porous Materials Center (AMPMC), Physical Sciences and Engineering Division, Thuwal 23955-6900, Kingdom of Saudi Arabia.

$\S$ Core Labs, King Abdullah University of Science and Technology (KAUST), Thuwal 23955-6900, Kingdom of Saudi Arabia.

\section{Supporting Information}

Chemicals. Hydrobromic acid (HBr, 48 wt. \% in water, Aladdin), cesium acetate ( $\geq 99.99 \%$, SigmaAldrich), manganese(II) acetate tetrahydrate (99.99\%, Sigma-Aldrich), manganese (II) bromide (98\%, Sigma-Aldrich), cesium bromide (99.999\%, Sigma-Aldrich), 1-octadecene (ODE, technical grade 90\%, Sigma-Aldrich), oleic acid (OA, 90\%, Alfa Aesar), n-oleylamine (OLA, 70\%, Sigma-Aldrich), toluene (Honeywell Burdick \& Jackson), benzoyl bromide (97\%, Sigma-Aldrich), ethyl acetate (99\%, Sigma-Aldrich), hexane (99\%, Alfa Aesar). Deionized water was used as solvent to prepare the single crystals. All chemicals were used as received without further purification.

Synthesis of $\mathrm{CsMnBr}_{3}$ nanocrystals. $\mathrm{CsMnBr}_{3}$ nanocrystals were synthesized by modifying a colloidal hot-injection approach developed for other lead-free metal halide nanocrystals (NCs). ${ }^{1} 0.4$ mmol of cesium acetate and $0.8 \mathrm{mmol}$ of manganese acetate tetrahydrate were transferred to a $100 \mathrm{~mL}$ round bottom reaction flask and mixed with $4 \mathrm{~mL}$ of ODE, $2 \mathrm{~mL}$ of OLA, and $2 \mathrm{~mL}$ of OA. The reaction mixture was degassed on Schlenk line for at least $4-5$ hours at $130{ }^{\circ} \mathrm{C}$. The temperature was then increased to $170{ }^{\circ} \mathrm{C}$ under nitrogen atmosphere. Once the temperature stabilized at $170{ }^{\circ} \mathrm{C}, 450$ $\mu \mathrm{L}$ of benzoyl bromide diluted in $1 \mathrm{~mL}$ of degassed ODE was swiftly injected into the reaction mixture. Immediately pinkish colored dispersion of NCs appeared and the reaction was quenched within 30 seconds using an ice-water bath. As-obtained crude dispersion of NCs is rotated at $6000 \mathrm{rpm}$ for 8 minutes and the precipitate was collected for further washing after discarding the supernatant. The precipitate was dispersed in $2 \mathrm{~mL}$ of hexane and $50 \mu \mathrm{L}$ of additional oleic acid was added to the 
colloidal dispersion of NCs before adding antisolvent. The NCs dispersion was destabilized by adding $\sim 6 \mathrm{~mL}$ of ethyl acetate followed by centrifugation at $6000 \mathrm{rpm}$ for 5 minutes. The precipitate was dispersed again in $2 \mathrm{~mL}$ of hexane after discarding supernatant. If oleic acid was not added, colloidal stability after $2^{\text {nd }}$ washing would be poor. A third washing was carried out similar to the second washing using additional $50 \mu \mathrm{L}$ of oleic acid. The final precipitate after three washings was dispersed in $2 \mathrm{~mL}$ hexane/toluene. By performing the reaction at a relatively lower temperature of $120{ }^{\circ} \mathrm{C}$, we were able to obtain smaller $\sim 9.5 \mathrm{~nm}$ sized $\mathrm{CsMnBr}_{3} \mathrm{NCs}$ (see Figure S8 for details on structure and optical properties of these NCs).

Synthesis of $\mathrm{CsMnBr}_{3}$ single crystals. The phase-pure single crystals were prepared with a typical [0.5:1] molar ratio of [ $\mathrm{CsBr}_{\mathrm{BnBr}} \mathrm{MnB}_{2}$. The solution was prepared with an adequate amount of deionized water and a small volume of $\mathrm{HBr}$ (5\% volume ratio of the original solution) added to obtain clear solution. The solution was kept at $40{ }^{\circ} \mathrm{C}$ in uncapped vial for few days in ambient conditions, and the crystals started appearing in 3 to 4 days. The crystals were then washed with anhydrous toluene to remove any leftover solution and dried with nitrogen flux. The synthesis was carried out in a relative humidity $(\mathrm{RH})$ of $60 \%$.

The powder X-ray diffraction. The powder X-ray diffraction (XRD) was performed on a Bruker AXS D8 diffractometer using $\mathrm{Cu}-\mathrm{K} \alpha$ radiation. The samples were prepared by drop casting colloidal dispersion of $\mathrm{CsMnBr}_{3} \mathrm{NCs}$ in hexane over glass substrate inside Ar-filled glovebox. Note that the films preserve their integrity when stored inside the glovebox. Exposure to humid atmosphere is minimized during the measurement by performing fast scan within 15 to 30 minutes.

Optical characterization. The optical absorption spectrum was measured using Cary 6000i UV-VisNIR spectrophotometer. PLQY and steady-state PL measurements were performed using an Edinburgh Instruments FLS920 Fluorescence Spectrometer equipped with $450 \mathrm{~W}$ continuous wavelength xenon lamp and integrating sphere.

Time-resolved photoluminescence (TRPL). Time-Resolved Photoluminescence measurements were obtained through the Time-Correlated Single-Photon counting technique (TCSPC). The excitation wavelengths $(380 \mathrm{~nm}, 450 \mathrm{~nm}$, and $520 \mathrm{~nm})$ were adjusted using a parametric optical amplifier (Newport, Spectra Physics) that was pumped with an Astrella femtosecond pulsed laser (800 nm, 100 fs, $1 \mathrm{kHz}$, Coherent). The energy at each excitation wavelength was set constant (34 $\mathrm{nJ}$ for solutions and $50 \mathrm{~nJ}$ for single crystals) with the help of variable neutral density filters (Thorlabs). The beam was directed into a Halcyone setup (Ultrafast Systems) where it was focused on the sample, and the monitored photoluminescence was collected and recollimated by a pair of parabolic mirrors and passed 
through a long-pass filter (540 nm, Newport) and lastly focused on the monochromator and detector. Solutions were measured in transmission mode, while single crystals were measured in the reflection mode. The obtained TCSPC histograms were fitted using Lavenberg-Marquart algorithm as implemented in Ultrafast System software. The overall system's time resolution was better than 120 ps.

Femtosecond transient absorption measurement. Femtosecond transient absorption (fs-TA) spectroscopy were performed on timescales of 0.1 ps to $6 \mathrm{~ns}$, which is based on a multipass amplified Ti:sapphire laser $(800 \mathrm{~nm}$ laser pulses of $7 \mathrm{~mJ} /$ pulse energy of $\sim 100 \mathrm{fs}$ pulse width having $1 \mathrm{kHz}$ repetition rate, Astrella from Coherent), and in conjunction with Helios spectrometers. The excitation pump pulses at 380 and $480 \mathrm{~nm}$ were generated after passing through a fraction of $800 \mathrm{~nm}$ beam into the spectrally tunable $(240-2600 \mathrm{~nm})$ optical parametric amplifier (Newport Spectra-Physics). The pump fluence $\left(0.5 \mu \mathrm{J} \mathrm{cm}^{-2}\right)$ of the excitation laser source was adjusted by using neutral density (ND) filter to avoid the multiple charge carriers generation. The probe pulses (UV visible and NIR wavelength continuum, white light) were generated by passing another fraction of the $800 \mathrm{~nm}$ pulses through the 2-mm thick calcium fluoride $\left(\mathrm{CaF}_{2}\right)$ crystal. Before white light generation the $800 \mathrm{~nm}$ amplified pulses were passed through a motorized delay stage. Depending on the movement of delay stage, the transient species were detected following excitation at different time scales. The white light was split into two beams (named as signal and reference) and focused on two fiber optics for the improvement of better signal to noise ratio. The excitation pump pulses were spatially overlapped with the probe pulses on the samples after passing through a synchronized mechanical chopper $(500 \mathrm{~Hz})$ which blocked an alternative pump pulses. The absorption change $(\Delta \mathrm{A})$ was measured with respect to the time delay and wavelength $(\lambda)$. All spectra were averaged over a time period of $2 \mathrm{~s}$ for each time delay.

Computational methods. Density functional theory (DFT) calculations were performed to optimize the crystal structures of $\mathrm{CsMnBr}_{3}, \mathrm{Cs}_{2} \mathrm{MnBr}_{4}$, and $\mathrm{Cs}_{3} \mathrm{MnBr}_{5}$ using the projector augmented wave (PAW) method as implemented in the VASP code. ${ }^{2-3}$ The Generalized Gradient Approximation (GGA)/Perdew-Burke-Ernzerhof (PBE) was used. The experimental crystal structures at room temperature were used as starting points and further optimized by relaxing both crystal lattices and atomic positions. The plane-wave cutoff energy was set to $500 \mathrm{eV}$ and a $\Gamma$-centered $6 \times 6 \times 6 \mathrm{k}$-mesh in the Brillouin zone were employed for bulk $\mathrm{CsMnBr}_{3}, \mathrm{Cs}_{2} \mathrm{MnBr}_{4}$, and $\mathrm{Cs}_{3} \mathrm{MnBr}_{5}$ see Figure $\mathrm{S} 7$ in this supporting information. All the crystal structures were further optimized under different external electric fields until the total force on each atom was $<0.01 \mathrm{eV} / \AA ̊$. The molecular graphics viewer (VESTA) was used to plot all the crystal structures. 

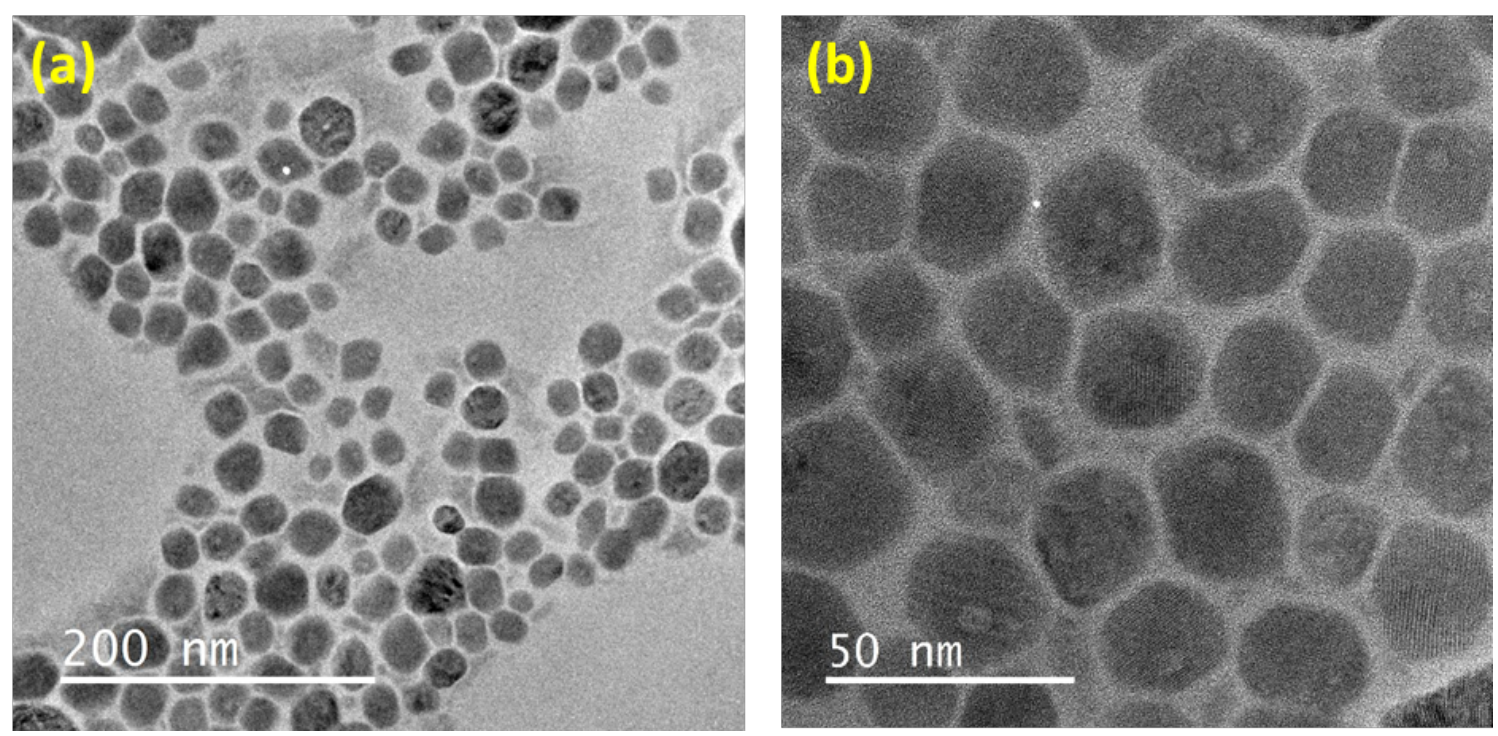

Figure S1. (a) Additional TEM images of $\mathrm{CsMnBr}_{3} \mathrm{NCs}$ with an average size of $25 \pm 4.8 \mathrm{~nm}$ along the elongated hexagonal shape (see size distribution in Figure 1g of main manuscript). (b) HRTEM image of NCs displaying lattice fringes.

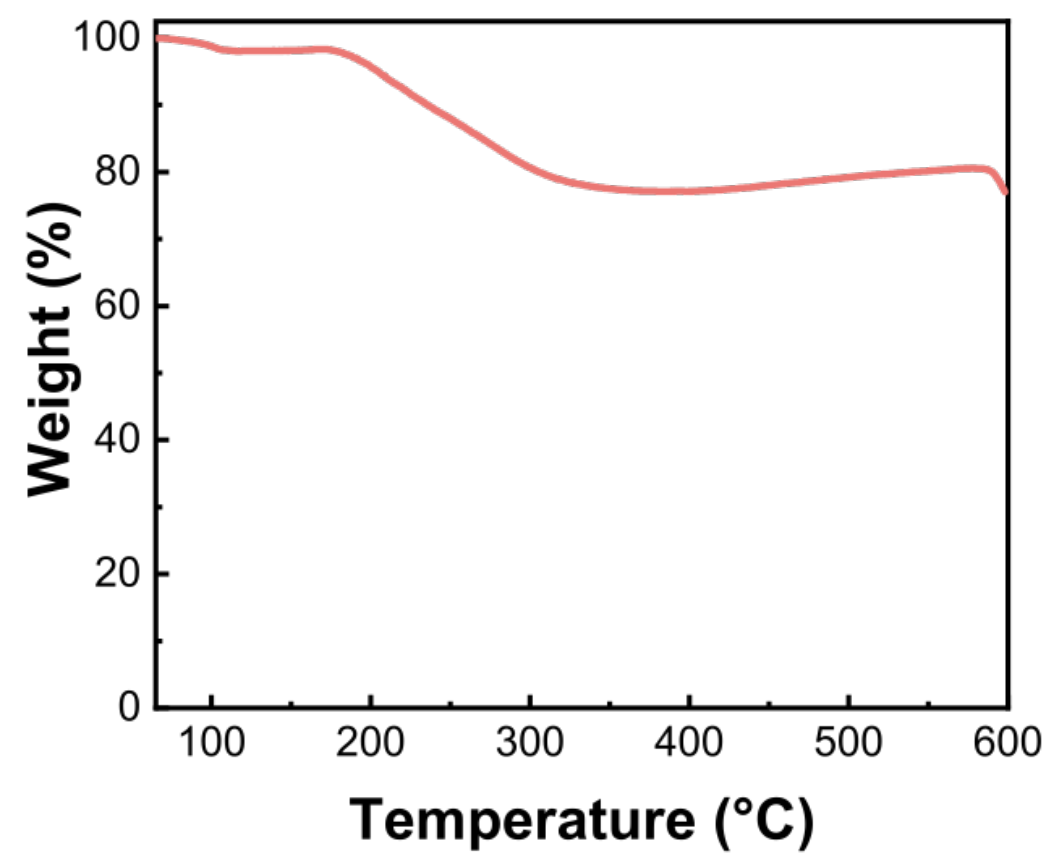

Figure S2. Thermogravimetric analysis of $\mathrm{CsMnBr}_{3} \mathrm{NCs}$ showing $21 \%$ weight loss due to the decomposition of organic ligands in the temperature range from 200 to $300{ }^{\circ} \mathrm{C}$. Few percent weight loss is observed around $100{ }^{\circ} \mathrm{C}$, which is due to solvent evaporation. 


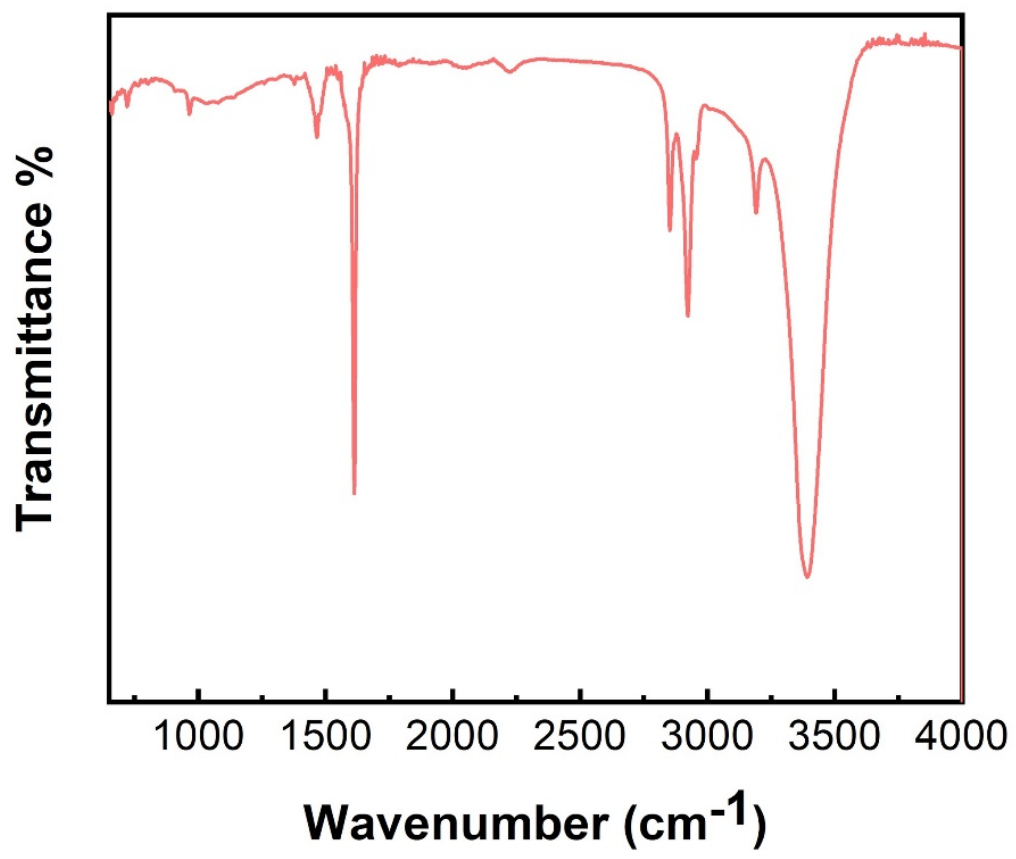

Figure S3. Fourier transform infrared (FTIR) spectra of $\mathrm{CsMnBr}_{3} \mathrm{NCs}$ that are capped with oleic acid and oleylamine.
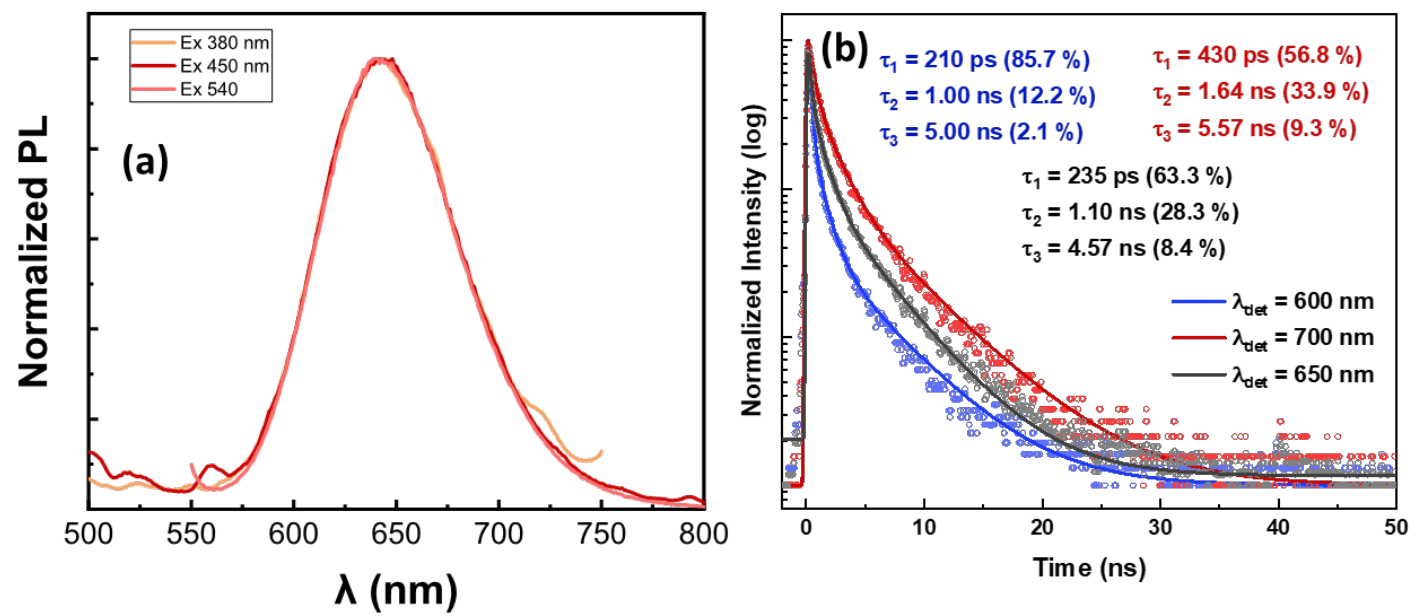

Figure S4. (a) PL emission spectra of $\mathrm{CsMnBr}_{3} \mathrm{NCs}$ after illuminations at three different excitation wavelengths, with identical spectral shape and FWHM. (b) Time-resolved PL decay dynamics of $\mathrm{CsMnBr}_{3} \mathrm{NCs}$ recorded at different emission wavelengths and fitted to a tri-exponential decay function; $\lambda_{\mathrm{exc}}=380 \mathrm{~nm}$. Qualitatively the nature of the PL decay dynamics remains similar at different emission energies suggesting a shared origin of emission. 


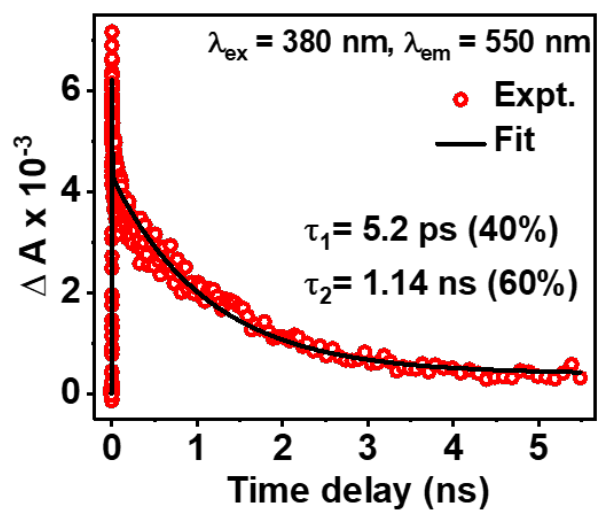

Figure S5. Transient kinetics as a function of time delay at $550 \mathrm{~nm}$ when excited at $380 \mathrm{~nm}$. The solid black line is a bi-exponential fitting of the experimental data (red symbols). The excited state decays to ground state with two components, $5.2 \mathrm{ps}(40 \%)$ and $1.14 \mathrm{~ns}(60 \%)$.
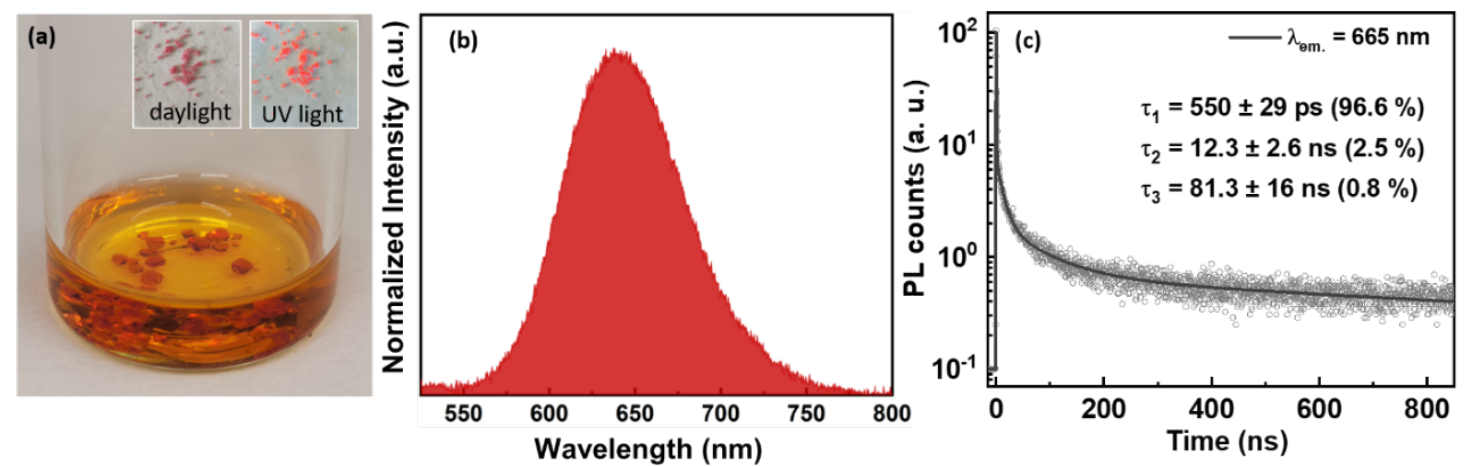

Figure S6. (a) Photograph of a vial containing $\mathrm{CsMnBr}_{3}$ single crystals obtained via , inset show photographs of single crystals obtained under day light and UV light. (b) PL spectra of CsMnBr 3 single crystal centred at $\sim 640 \mathrm{~nm}$ with full-width half maximum of $\sim 80 \mathrm{~nm}$. The single crystals show PLQY $\sim 6.7 \%$ when measured immediately after synthesis. Qualitatively red PL emission from single crystal is similar to PL emission from NCs. (c) Time-resolved PL decay dynamics of $\mathrm{CsMnBr}_{3}$ single crystal fitted with a tri-exponential decay function. The fitted components obtained are $550 \mathrm{ps}(96.6 \%), 12.3$ ns $(2.5 \%)$, and $81.3 \mathrm{~ns}(0.8 \%) ; \lambda_{\mathrm{exc}}=380 \mathrm{~nm}$.

$\mathrm{CsMnBr}_{3}$

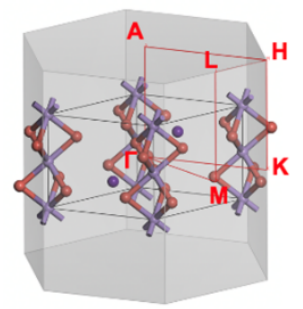

$\mathrm{Cs}_{2} \mathrm{MnBr}_{4}$

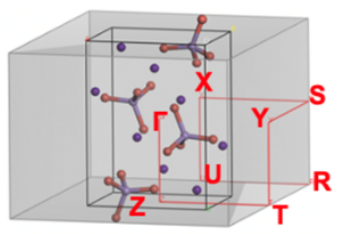

$\mathrm{Cs}_{3} \mathrm{MnBr}_{5}$

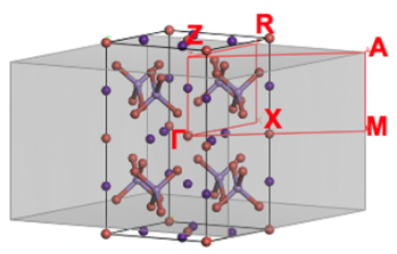

Figure S7. $\Gamma$-centered $6 \times 6 \times 6 \mathrm{k}$-mesh of the Brillouin zones that were probed using DFT in $\mathrm{CsMnBr}_{3}$, $\mathrm{Cs}_{2} \mathrm{MnBr}_{4}$, and $\mathrm{Cs}_{3} \mathrm{MnBr}_{5}$. 

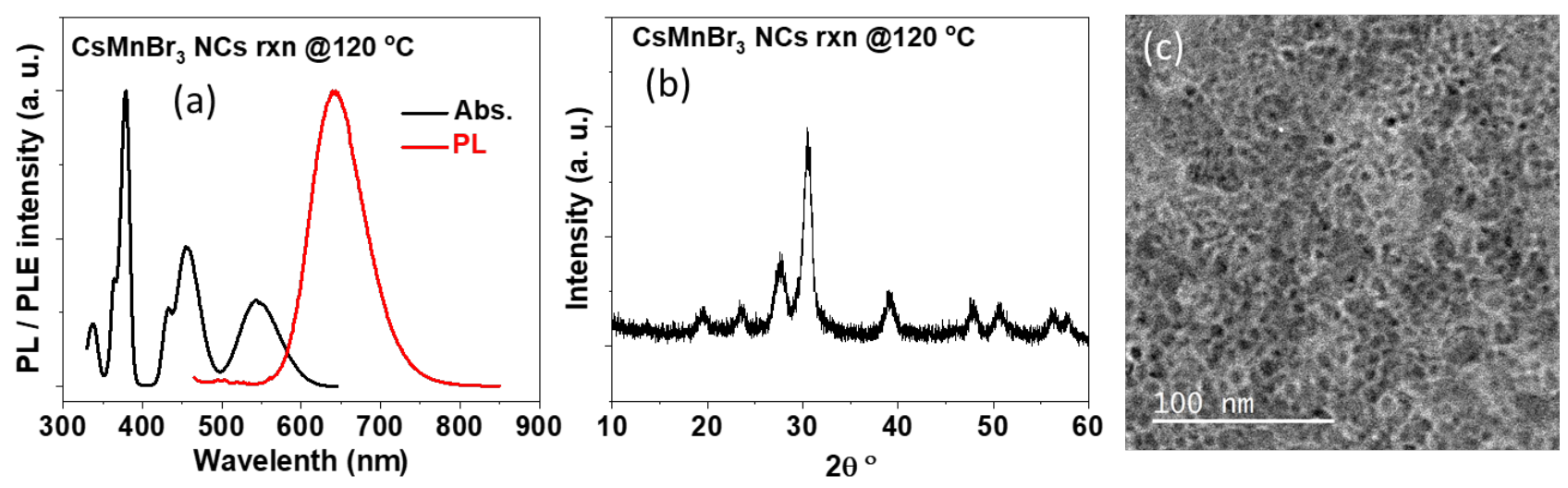

Figure S8. Optical and structural properties of small sized $\mathrm{CsMnBr}_{3} \mathrm{NCs}$ that are obtained by performing reaction at low temperature of $120{ }^{\circ} \mathrm{C}$. (a) PL and PLE spectra of NCs dispersed in anhydrous toluene. (b) XRD pattern of NCs forming hexagonal phase and with an average Debye Scherer size of $10 \mathrm{~nm}$. (c) TEM image of $\mathrm{CsMnBr}_{3} \mathrm{NCs}$ with an average size of $9.5 \pm 0.4 \mathrm{~nm}$. By performing the colloidal synthesis of $\mathrm{CsMnBr}_{3} \mathrm{NC}$ at a relatively low temperature of $120^{\circ} \mathrm{C}$, we were able to obtain smaller NCs $(\sim 9.5 \mathrm{~nm})$. The PLE and PL are qualitatively similar to the larger NCs ( $25 \mathrm{~nm}$ ) that are obtained at $170{ }^{\circ} \mathrm{C}$ (see the main manuscript for details), but the PLQY of the smaller NCs decreased to $42 \%$ compared to $54 \%$ for the larger NCs. The absence of a PL and PLE shift in the smaller $\mathrm{CsMnBr}_{3} \mathrm{NCs}$ compared to the larger $\mathrm{NCs}$ is expected since the optical transitions originating from the d-d transition of Mn are usually unaffected by the crystal size. 
Table S1. Single crystal X-ray diffraction details of $\mathrm{CsMnBr}_{3}$ single crystal.

\begin{tabular}{|c|c|}
\hline Chemical formula & $\mathrm{Br}_{3} \mathrm{CsMn}$ \\
\hline$M_{\mathrm{r}}$ & 427.58 \\
\hline Crystal system, space group & Hexagonal, $\mathrm{P}_{3} / \mathrm{mmc}$ \\
\hline Temperature (K) & 120 \\
\hline$a, b, c(\AA)$ & $7.5593(10), 6.4740(11)$ \\
\hline$V\left(\AA^{3}\right)$ & $320.38(10)$ \\
\hline$Z$ & 2 \\
\hline Radiation type & $\operatorname{MoK} \alpha(\lambda=0.71073)$ \\
\hline$\mu\left(\mathrm{mm}^{-1}\right)$ & 26.219 \\
\hline Crystal size (mm) & $0.3 \times 0.3 \times 0.25$ \\
\hline Diffractometer & Bruker APEX-II CCD \\
\hline Absorption correction & Multi-scan \\
\hline \multicolumn{2}{|l|}{$T_{\min }, T_{\max }$} \\
\hline $\begin{array}{l}\text { No. of } \text { measured, } \\
\text { independent and } \\
\text { observed }[I>2 \sigma(I)] \\
\text { reflections }\end{array}$ & $4468,192,241$ \\
\hline$R_{\text {int }}$ & 0.0658 \\
\hline$(\sin \theta / \lambda)_{\max }\left(\AA^{-1}\right)$ & 0.67 \\
\hline$R\left[F^{2}>2 \sigma\left(F^{2}\right)\right], w R\left(F^{2}\right), S$ & $\begin{array}{c}\mathrm{R}_{1}=0.0234, \mathrm{wR}_{2}=0.0599 \\
\mathrm{~S}=1.200\end{array}$ \\
\hline No. of reflections & 4468 \\
\hline No. of parameters & 10 \\
\hline$\Delta \rho_{\max }, \Delta \rho_{\min }\left(\mathrm{e} \AA^{-3}\right)$ & $1.99 /-1.02$ \\
\hline
\end{tabular}


Table S2. PL decay parameters of $\mathrm{CsMnBr}_{3}$ NCs fitted with a tri-exponential decay function from the experimental data shown in Figure 3a of the main manuscript for three different excitation wavelengths.

\begin{tabular}{|c|c|c|c|}
\hline$\lambda$ (excitation) & $\boldsymbol{\tau}_{\mathbf{1}}$ & $\boldsymbol{\tau}_{\mathbf{2}}$ & $\boldsymbol{\tau}_{\mathbf{3}}$ \\
\hline $380 \mathrm{~nm}$ & $\begin{array}{c}653.4 \pm 44 \mathrm{ps} \\
(67.7 \%)\end{array}$ & $\begin{array}{c}2.0 \pm 0.11 \mathrm{~ns} \\
(27.4 \%)\end{array}$ & $\begin{array}{c}12.9 \pm 0.73 \mathrm{~ns} \\
(4.9 \%)\end{array}$ \\
\hline $450 \mathrm{~nm}$ & $\begin{array}{c}549.4 \pm 67 \mathrm{ps} \\
(91.6 \%)\end{array}$ & $\begin{array}{c}1.7 \pm 0.28 \mathrm{~ns} \\
(5.6 \%)\end{array}$ & $\begin{array}{c}20.8 \pm 1.1 \mathrm{~ns} \\
(2.72 \%)\end{array}$ \\
\hline $540 \mathrm{~nm}$ & $605.0 \pm 56 \mathrm{ps}$ & - & - \\
\hline
\end{tabular}

\section{References}

1. Zhu, D.; Zaffalon, M. L.; Pinchetti, V.; Brescia, R.; Moro, F.; Fasoli, M.; Fanciulli, M.; Tang, A.; Infante, I.; De Trizio, L.; Brovelli, S.; Manna, L., Bright Blue Emitting Cu-Doped $\mathrm{Cs}_{2} \mathrm{ZnCl}_{4}$ Colloidal Nanocrystals. Chem. Mater. 2020, 32, 5897-5903

2. Kresse, G.; Hafner, J., Ab initio molecular dynamics for open-shell transition metals. Phys. Rev. $B$ 1993, 48, 13115-13118.

3. Kresse, G.; Furthmüller, J., Efficient iterative schemes for ab initio total-energy calculations using a plane-wave basis set. Phys. Rev. B 1996, 54, 11169-11186. 